\title{
Renal function tests in patients with Cutaneous Leishmania after treatment with Glucantime
}

\author{
Ibrahim W. Hasani, Abdul-Razak Kotayni, Samer kabba
}

\begin{abstract}
Cutaneous leishmaniasis (CL) has historically been present in Syria, mainly in the western part of the country. The present study was conducted to evaluate kidney function parameters in patients infected with $C L$ in Idlep-Syria. This is controlled study contain infected (60) and control (30) groups. The prevalence and distribution of infection were studied, kidney function testes were determined by colorimetric methods, $\left(\right.$ Biosystem $\left.{ }^{\circledR}\right)$. Among 100 of a total cases studied, the percent of infected cases are $60 \%$ and not infected are $40 \%$. The distribution of lesions on various parts of body in patients shows the highest percent of infection was in face $(50 \%)$. There were highly significant increase in urea, uric acid and creatinine in infected group ( $p<0.0001)$ as compared to control one. In the war in Syria, the prevalence of infection was increased and had side effect on kidney parameter in infected patients treated with Glucantime.
\end{abstract}

Key Words: Cutaneous leishmaniasis (CL), Idlep-Syria, kidney function tests, Glucantime

\section{INTRODUCTION}

Leishmaniasis is a poverty-related disease with two main clinical forms: visceral leishmaniasis and cutaneous leishmaniasis. An estimated 0.7-1 million new cases of leishmaniasis per year are reported from nearly 100 endemic countries. The number of reported cutaneous leishmaniasis cases has increased dramatically in the past few years as a result of Increased conflict in endemic areas of cutaneous leishmaniasis and forced displacement has resulted in a surge in these endemic areas. WHO lists leishmaniasis as one of the neglected tropical diseases for which the development of new treatments is a priority. Major evidence gaps remain, and new tools are needed before leishmaniasis can be definitively controlled [1].

Cutaneous leishmaniasis (CL) has historically been present in Syria, mainly in the western part of the country [2]. Anthroponotic CL due to Leishmania tropica is the most common form in the country, but zoonotic CL due to L. major has also been reported [3]. The overall health situation in Syria has been heavily reshaped by the war

Ibrahim W. Hasani, Department of Biochemistry, Faculty of Science, Idlip University, Syria

Abdul-Razak Kotayni, Department of Biochemistry, Faculty of Science, Idlip University, Syria.

Samer kabba, Department of pharmaceutics, Faculty of pharmacy, Northern Private University(NPU), Syria. (2011-present), due to the deterioration of healthcare infrastructures, challenges in the implementation of control measures, and mass-scale displacement of populations, both within Syria and to other countries (6.2 million internally displaced persons [IDPs] and 5.7 million registered refugees as of March 2019) [4].

This can be explained by the fact that the war has also negatively impacted the CL surveillance and reporting system. While up until the onset of hostilities the $\mathrm{MoH}$ routine surveillance system covered the entire national territory, since 2011 the changing frontline between fighting entities has meant that only facilities in areas controlled by the government, and therefore still run by $\mathrm{MoH}$ staff, could report to the central units in Damascus, while the freedom area like Idlep don't have any surveillance system.

(Glucantime) is a pentavalent antimony $\left(\mathrm{Sb}^{\mathrm{V}}\right)$ recommended by the World Health Organization as the first-choice drug for the treatment of all types of leishmaniasis; the maximum dose recommended is $20 \mathrm{mg} / \mathrm{kg}$ of body weight/day via the intramuscular route. Although pentavalent antimonials have been used for many years, the pharmacological and toxicological mechanisms involved in their action remain unclear [5]. Berman et al. [6] and Demicheli et al. [7] proposed that this drug interferes with the bioenergetic process of Leishmania amastigotes, which inhibit parasites fatty acid -oxidation and glycolysis, promoting the depletion of intracellular ATP levels. Another hypothesis suggests that $\mathrm{SbV}$ acts as a prodrug that is transformed into the more toxic trivalent form (SbIII) to exert its antileishmanial activity [8]. Unfortunately, many side effects are still reported. The most frequently reported clinical adverse effects are musculoskeletal pain, nausea, vomiting, diarrhea, abdominal pain, headache, anorexia, asthenia, fatigue, fever, exanthema, erythema, and urticaria. Moreover, patients may develop cardiac, liver, and kidney complications, usually at the end of the treatment [9].

The war coincided with a major rise in the reported number of CL cases and incidence of infection, although an increasing trend was already appreciable before its onset. Therefore, the present study was conducted to study the effect of CL treated with Glucantime on kidney function tests. 


\section{METHODS AND PATIENTS}

\section{Patients}

This is a control study with 60 patients with confirmed diagnosis of CL, between March and August 2018 in compared with control group. In the present study there was no significant deference between two group in relation to age ( $p>0.05)$. Exclusion criteria was patients with hypertension, diabetes mellitus, urinary tract infection kidney disease. The 60 patients were compared with 30 healthy volunteers. The protocol of this study was approved by the Ethical Comitee of Idlep University. Patients were included in the study after signing the informed consent form.

\section{Diagnosis of $\mathbf{C L}$}

Diagnosis of CL was based on epidemiologic and clinical criteria. Cases were collected at Maarshorin and Khan-shikhon Center in Idlep- Syria. Diagnosis was confirmed clinically, as well as by laboratory demonstration of the parasite in the lesions by direct smears using microscopic examination as mentioned early by[10], briefly, tissue scrapings performed with staining the specimen with by Giemsa stain. The lesions cleaned and any eschars or exudates was removed. $1 \%$ lidocaine used to decrease bleeding, optimize debridement, and obtain and improve tissue scraping quality. For tissue scrapings, ten scalpel blade was used. Scraping was performed with a pressure that is adequate to obtain exudates, without evoke bleeding. The dermal tissue was spread in a $2-3 \mathrm{~cm}$ diameter on a glass slide then fixed briefly with methanol, Giemsa stained, and examined for the presence of amastigotes.

$10 \mathrm{ml}$ of venous blood were collected from 60 patients as well as from 30 control after an overnight fast and it was positive. The aspirated blood was immediately put into two different test tube, the other fraction of blood was clotted in plain test tube, then the serum was separated by centrifugation at $2000 \times g$ and used for the quantitation of the biochemical reaction. At the time of medical consult all symptoms and signals were evaluated, as well as age, gender, , number of skin lesions, time of disease and use of drugs. The following laboratory tests were studied in serum: urea, creatinine and uric acid.

All patients underwent standard treatment with Glucantime in the dose of $20 \mathrm{mg} / \mathrm{kg} /$ day for 20 days. All tests were done after treatment.

\section{Analytical methods}

Serum uric acid was determined by colorimetric uricase method $\left(\right.$ Biosystem ${ }^{\circledR}$ ). The results were expressed in $\mathrm{mg} / \mathrm{dl}$. Serum creatinine: Determined by colorimetric methods, picric acid, (Biosystem ${ }^{\circledR}$ ). The results were expressed in $\mathrm{mg} / \mathrm{dl}$. Serum urea: Determined by colorimetric reaction $\left(\right.$ Biosystem $\left.^{\circledR}\right)$. The results were expressed in $\mathrm{g} / \mathrm{dl}$.

\section{Statistical analysis:}

All quantitative data are expressed as mean $\pm \mathrm{SEM}$. Differences between two parameters were analyzed either by paired Student $t$ test or by nonparametric methods (Wilcoxon test and Mann-Whitney test). Values of $\mathrm{P}<0.05$ were considered statistically significant

\section{A. Results}

\section{1) Epidemiological study}

a)

$$
\begin{aligned}
& \text { Prevalence of cutaneous leishmaniasis } \\
& \text { infection }
\end{aligned}
$$

Among 100 of a total cases studied, the percent of infected cases are $60 \%$ and not infected are $40 \%$. Most prevalence rate which were positive collected from Maarshorin district with percent to $(67.5 \%)$, while the little less percent in Khan-shekhone (55\%).

\section{Types and distribution of lesions}

Distribution of lesions on various parts of body in patients shows the highest percent of infection was in face (50\%), and the lowest on the legs and feet (3.3 and $13.3 \%$ respectively) (Fig. 1) and table (1).

Table (1): Distribution of cutaneous leishmaniasis among examined cases, according to the site of infection

\begin{tabular}{|c|c|}
\hline Site of infection & Number(\%) \\
\hline Face & $30(50 \%)$ \\
\hline Hands & $20(33.3 \%)$ \\
\hline Feet & $8(13.3 \%)$ \\
\hline Legs & $2(3.3 \%)$ \\
\hline
\end{tabular}

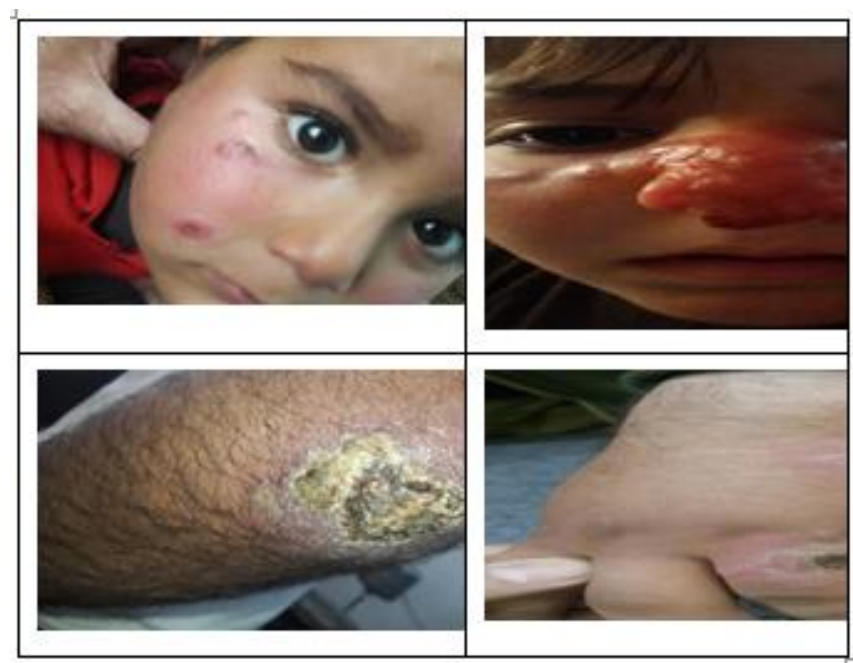

Figure (1): Pictures of lesions in patients with CL 


\section{Kidney function tests:}

In the present study there were significant increase in uric acid, urea and creatinine in infection group as compared to control group $(\mathrm{P}<0.0001)$ separately fig. (2).

\section{A}
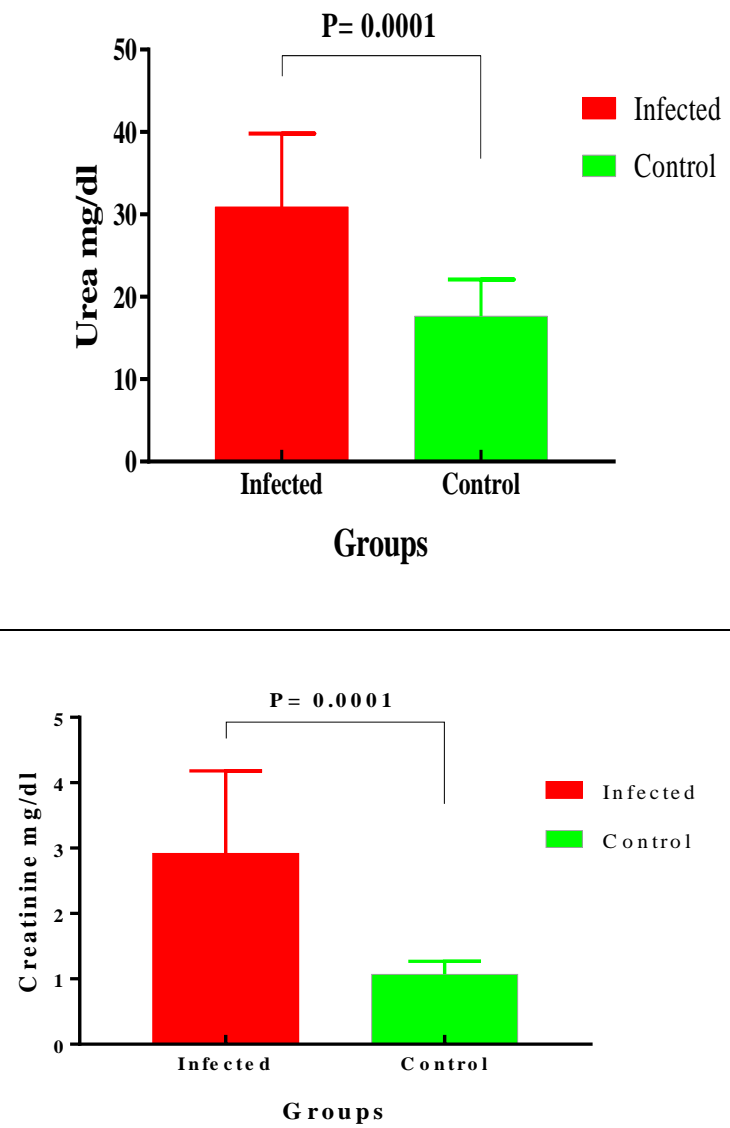

B

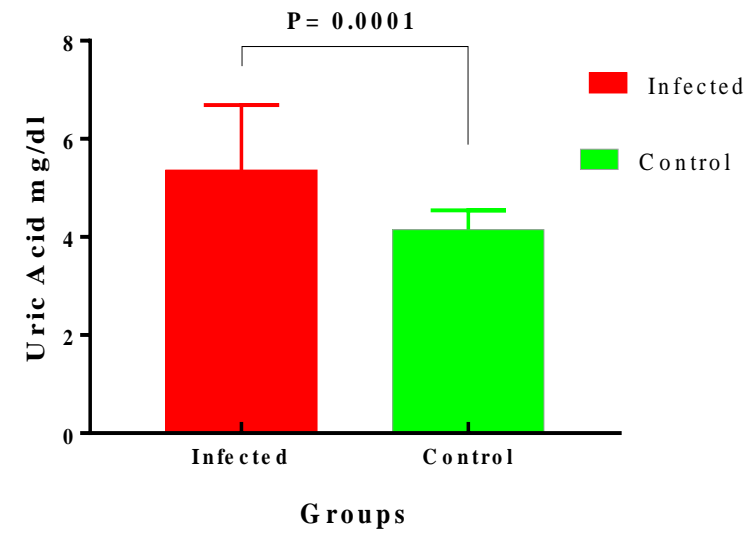

C

Figure (2): Kidney function testes (A: Urea, B: Creatinine and C: Uric Acid) in the studied group

\section{Discussion}

The results of the present study evidence the occurrence of kidney function abnormality probably induced by CL and treated with Glucantime.

In the present study, the single lesions are observed in most of the patients. The cause of differences of scars type maybe due to the variation of the vectors. The same discussion explained by [11],present study does not cover the vector. Cutaneous leishmaniasis in Idlep-Syria is caused by Leishmania. The vectors are sand flies of the genus Phlebotomies. The incubation period ranges from weeks to months. Present study shows some lesions appeared typically on exposed areas of the body where inoculation occurs. The same scars have been founded by [12]. Lesions appear as small nodules, or round with raised margins and a granulating center with yellowish exudates which increase in size and eventually ulcerate, that depend on parasite, host, and sand fly factors; dose or route of inoculation; and the maintenance of macrophages in an inert, deactivated state [13].

Our study shows that uric acid, creatinine and urea levels have high significant $(\mathrm{P}<0.0001)$ increase in patients groups than those of control groups. Uric acid is an important contributor to total antioxidant capacity; it provides a significant antioxidant defense against nitration by proxy nitrite. It has an important role as an oxidative stress marker and a potential therapeutic role as an antioxidant [14].

In a study involving 11 patients with ACL treated with pentavalent antimonial, $40 \mathrm{mg} / \mathrm{kg}$ for 30 days, the persisting urinary concentrating deficit was observed in 8 cases $(72.7 \%)$ [15]. Veiga et al. [16],reported 5 cases of ACL treated with pentavalent antimonial in conventional doses, but with a longer duration, which developed urinary concentrating inability. Lima Verde et al. [17], found $68 \%$ of urinary concentrating capacity defect in patients with kala-azar before pentavalent antimonial therapy. The persistence of this abnormality can be a consequence of CL itself, which can cause a severe tubular damage.

This study shows that there is a high significant $(\mathrm{P}<0.001)$ increase of the uric acid level in patients groups than those of controls. The same result was reported by [18]. This increase in uric acid level which may refer to the physiological activity and the influence of destroyed or catabolism [19].Increased level of uric acid may contribute much more to scavenging of free radicals. This may support the powerful antioxidant role of uric acid in scavenging singlet oxygen and other free radicals [20] and [21]. Uric acid may act as a defense mechanism against oxidative stress, or uric acid acting as a pro-oxidant and contributing to the damage caused in these diseases [22]. Uric acid is released from tissues that are short of oxygen and elevated uric acid levels may an important part of acclimatization to high altitude [23].

L-arginine can be oxidized by host inducible nitric oxide synthase (iNOS) to produce nitric oxide (NO), which contributes to parasite killing. In contrast, L-arginine 
hydrolysis by host arginase blocks NO generation and provides polyamines, which can support parasite proliferation. Arginase hydrolyzes L-arginine to form urea and ornithine [24], which explain in partially of increasing of urea in infected group. Previous reports on the renal involvement in CL have linked renal abnormalities to the use of drugs (Glucantime), as acute kidney injury by tubule interstitial nephritis [25][26].

The present study, showed clearly that infected patients treated with Glucantime led to kidney abnormality. Further studies are required to better understand the mechanisms involved in kidney dysfunction caused by $\mathrm{CL}$ and by the treatment. Additionally, Further investigations, with a longer period of observation after CL treatment, would be important to establish the long-term outcomes regarding this complication.

\section{References:}

[1] S. Burza, S. L. Croft, and M. Boelaert, "Leishmaniasis," Lancet, vol. 392, no. 10151, pp. 951-970, Sep. 2018.

[2] G. Muhjazi et al., "Cutaneous leishmaniasis in Syria: A review of available data during the war years: 2011-2018," PLoS Negl. Trop. Dis., vol. 13, no. 12, p. e0007827, Dec. 2019.

[3] J.-A. Rioux, J. Dereure, A. Khiami, F. Pratlong, K. Sirdar, and M. Lambert, "Ecoépidémiologie des leishmanioses en Syrie. 1 - Infestation de Psammomys obesus Cretzschmar (Rodentia-Gerbillidae) par Leishmania major Yakimoff et Schokhor (Kinetoplastida-Trypanosomatidae)," Ann. Parasitol. Hum. Comparée, vol. 65, no. 5-6, pp. 203-207, Aug. 1990.

[4] S. Devi, "Syria: 7 years into a civil war," Lancet, vol. 391, no. 10115, pp. 15-16, Jan. 2018.

[5] V. R. Moreira et al., "Meglumine Antimoniate (Glucantime) Causes Oxidative Stress-Derived DNA Damage in BALB/c Mice Infected by Leishmania (Leishmania) infantum," Antimicrob. Agents Chemother., vol. 61, no. 6, Jun. 2017.

[6] J. D. Herman, J. V. Gallalee, and J. M. Best, "Sodium stibogluconate (pentostam) inhibition of glucose catabolism via the glycolytic pathway, and fatty acid $\beta$-oxidation in leishmania mexicana amastigotes," Biochem. Pharmacol., vol. 36, no. 2, pp. 197-201, Jan. 1987.

[7] C. Demicheli, F. Frézard, M. Lecouvey, and A. Garnier-Suillerot, "Antimony(V) complex formation with adenine nucleosides in aqueous solution," Biochim. Biophys. Acta - Gen. Subj., vol. 1570, no. 3, pp. 192-198, Apr. 2002.

[8] N. Miekeley, S. Mortari, and A. Schubach, "Monitoring of total antimony and its species by ICP-MS and on-line ion chromatography in biological samples from patients treated for leishmaniasis," Anal. Bioanal. Chem., vol. 372, no. 3, pp. 495-502, Feb. 2002.

[9] V. R. Moreira et al., "Meglumine antimoniate (glucantime) causes oxidative stress-derived DNA damage in Balb/c mice infected by leishmania (leishmania) infantum," Antimicrob. Agents Chemother., vol. 61, no. 6, 2017.

[10] Q. Asmaa, S. AL-Shamerii, M. Al-Tag, A. AL-Shamerii, Y. Li, and B. H. Osman, "Parasitological and biochemical studies on cutaneous leishmaniasis in Shara'b District, Taiz, Yemen," Ann. Clin. Microbiol. Antimicrob., vol. 16, no. 1, p. 47, Dec. 2017.

[11] M. Kharfi et al., "Childhood leishmaniasis: report of 106 cases.," Dermatol. Online J., vol. 10, no. 2, p. 6, Oct. 2004.

[12] S. Mings, J. C. Beck, C. Davidson, A. L. Ondo, S. D. Shanler, and J. Berman, "Cutaneous leishmaniasis with boggy induration and simultaneous mucosal disease.," Am. J. Trop. Med. Hyg., vol. 80, no. 1, pp. 3-5, Jan. 2009.

[13] M. K. Basu and M. Ray, "Macrophage and Leishmania: An Unacceptable Coexistence," Crit. Rev. Microbiol., vol. 31, no. 3, pp. 145-154, Jan. 2005.

[14] R. .-J. Teng, Y.-Z. . Ye, D. A. Parks, and J. S. Beckman, "Urate produced during hypoxia protects heart proteins from peroxynitrite-mediated protein nitration," Free Radic. Biol. Med., vol. 33, no. 9, pp. 1243-1249, Nov. 2002.

[15] R. N. R. Sampaio et al., "Avaliação da tolerância e nefrotoxicidade do antimonial pentavalente administrado na dose de 40mg Sb v/kg/dia, de $12 / 12 \mathrm{~h}$, por 30 dias na forma cutaneo-mucosa de leishmaniose," Rev. Soc. Bras. Med. Trop., vol. 30, no. 6, pp. 457-463, Dec. 1997.

[16] J. P. R. Veiga et al., "Pentavalent antimonial nephrotoxicity in the rat," Rev. Inst. Med. Trop. Sao Paulo, vol. 32, no. 4, pp. 304-309, Aug. 1990.

[17] F. A. A. Lima Verde, F. A. Lima Verde, I. A. Lima Verde, G. B. Silva Junior, E. F. Daher, and E. M. Lima Verde, "Evaluation of renal function in human visceral leishmaniasis (kala-azar): a prospective study on 50 patients from Brazil.," J. Nephrol., vol. 20, no. 4, pp. 430-6, 2007.

[18] G. Glantzounis, E. Tsimoyiannis, A. Kappas, and D. Galaris, "Uric Acid and Oxidative Stress," Curr. Pharm. Des., vol. 11, no. 32, pp. 4145-4151, Dec. 2005.

[19] M. Mahajan, S. Kaur, S. Mahajan, and R. Kant, "Uric acid a better scavenger of free radicals than vitamin C in rheumatoid arthritis," Indian J. Clin. Biochem., vol. 24, no. 2, pp. 205-207, Apr. 2009.

[20] C.-C. Chuang et al., "Serum total antioxidant capacity reflects severity of illness in patients with severe sepsis.," Crit. Care, vol. 10, no. 1, p. R36, Feb. 2006.

[21] F. Bayiroğlu, M. Cemek, H. Çaksen, F. Cemek, and S. Dede, "Altered Antioxidant Status and Increased Lipid Peroxidation in Children with Acute Gastroenteritis Admitted to a Pediatric Emergency Service," J. Emerg. Med., vol. 36, no. 3, pp. 227-231, Apr. 2009.

[22] H. V. Dimitroula, A. I. Hatzitolios, and H. I. Karvounis, "The Role of Uric Acid in Stroke," Neurologist, vol. 14, no. 4, pp. 238-242, Jul. 2008.

[23] C. F. Araujo, M. V. Lacerda, D. S. Abdalla, and E. S. Lima, "The role of platelet and plasma markers of 
antioxidant status and oxidative stress in thrombocytopenia among patients with vivax malaria," Mem. Inst. Oswaldo Cruz, vol. 103, no. 6, pp. 517-521, Sep. 2008.

[24] H. M. Muleme et al., "Infection with Arginase-Deficient Leishmania major Reveals a Parasite Number-Dependent and Cytokine-Independent Regulation of Host Cellular Arginase Activity and Disease Pathogenesis," $J$. Immunol., vol. 183, no. 12, pp. 8068-8076, Dec. 2009.

[25] R. A. Oliveira et al., "Renal function evaluation in patients with American Cutaneous Leishmaniasis after specific treatment with pentavalent antimonial," BMC Nephrol., vol. 13, no. 1, p. 44, Dec. 2012.

[26] M. L. O. RODRIGUES, R. S. COSTA, C. S. SOUZA, N. T. FOSS, and A. M. F. ROSELINO, "NEPHROTOXICITY ATTRIBUTED TO MEGLUMINE ANTIMONIATE (GLUCANTIME) IN THE TREATMENT OF GENERALIZED CUTANEOUS LEISHMANIASIS," Rev. Inst. Med. Trop. Sao Paulo, vol. 41, no. 1, pp. 33-37, Jan. 1999. 